\title{
Analysis of Risk Factors of Recurrent Vertebral Fractures After Percutaneous Vertebroplasty
}

\author{
Jiang Du, Chenhuan Lu, Jing Wang* \\ Department of Orthopedics, the First Affiliated Hospital of Jinan University, Guangzhou, China \\ Email address: \\ twjing@jnu.edu.cn (Jing Wang) \\ ${ }^{*}$ Corresponding author \\ To cite this article: \\ Jiang Du, Chenhuan Lu, Jing Wang. Analysis of Risk Factors of Recurrent Vertebral Fractures After Percutaneous Vertebroplasty. Journal of \\ Surgery. Vol. 8, No. 6, 2020, pp. 204-208. doi: 10.11648/j.js.20200806.16
}

Received: November 1, 2020; Accepted: November 19, 2020; Published: November 30, 2020

\begin{abstract}
Background: Investigate the risk factors of recurrent vertebral compression fractures after percutaneous vertebroplasty (PVP) for osteoporotic vertebral compression fractures (OVCF). Method: Data of 401 patients with osteoporotic vertebral compression fractures treated by PVP from March 2013 to June 2018 were retrospectively analyzed, and were divided into re-fracture group and nonre-fracture group according to the postoperative recurrence of vertebral compression fractures. The following parameters were observed, including age, gender, bone mineral density (BMD), correction degree of kyphosis, recovery degree of vertebral height, amount of bone cement injected, Pfirrmann classification of adjacent intervertebral disc of hurt vertebral, and bone cement leakage in intervertebral disc. Then, the above parameters were statistically analyzed by univariate and multivariate analysis to explore the risk factors of vertebral recurrent fracture after PVP. Result: Among 401 patients, 34 (34/401, 8.4\%) recurred OVCF after PVP. Statistical analysis showed that the risk of recurrent vertebral fracture increased by 3.732 times (95\% CI 1.107-12.581) when Pfirrmann classification of adjacent intervertebral disc was in degeneration grade. The risk of recurrent vertebral fracture was significantly increased by 31.818 times $(95 \%$ CI 13.384-75.640) when bone cement leakage occurred in intervertebral disc. Conclusion: Pfirrmann classification of adjacent intervertebral disc and bone cement leakage in intervertebral disc are significantly correlated with the recurrence of vertebral fracture after PVP. In PVP operation, avoiding the bone cement leakage in intervertebral disc can significantly reduce the recurrence of vertebral compression fractures.
\end{abstract}

Keywords: Percutaneous Vertebroplasty, Osteoporotic Vertebral Compression Fractures, Recurrent Fractures, Risk Factors

\section{Introduction}

With the development of aging population in China, osteoporotic vertebral compression fracture is becoming a daily increasing serious public health problem. Percutaneous vertebroplasty (PVP) has been widely used in clinical practice because it can quickly relieve the pain symptoms in waist and back of patients and restore their life quality, and has gradually become the most commonly used method [1] for the treatment of osteoporotic vertebral compression fractures (OVCF). Although a large number of studies have confirmed its good clinical efficacy, and there is controversy that PVP will increase the risk [2] of surgery or recurrent fracture in adjacent vertebral body. There are also views think postoperative fracture has nothing to do with the operation itself, which is the natural course of osteoporosis [3]. In this paper, the characteristics and related factors of recurrent compression fractures after PVP were retrospectively analyzed to summarily analyze the risk factors and put forward prevention methods, to reduce the risk of recurrent fractures after PVP.

\section{Materials and Methods}

\subsection{General Materials}

All OVCF patients who were treated with PVP in the First Affiliated Hospital of Jinan University from March 2013 to June 2018 were retrospectively analyzed and grouped according to the following diagnostic criteria. The diagnostic criteria for recurrence fracture group (Group A) were as follows: (1) low back pain and movement disorder occurred again after obvious improvement or relief of postoperative 
pain; (2) X-ray and MR confirmed that there was a clear responsibility segment of fresh compression fracture, and this segment was not found abnormal in MR examination before the first PVP. The diagnostic criteria of the control group (group B) without recurrence fracture were as follows: (1) the postoperative pain was relieved and no recurrence symptom; (2) the postoperative X-ray examination showed that the vertebral height was not significantly compressed. Then the above cases were screened according to the following exclusion criteria: (1) incomplete imaging or follow-up data; (2) secondary to severe trauma, tumor, hemangioma, and other non-osteoporosis vertebral fractures. After screening, 401 patients were included, including 34 cases in group A and 367 cases in group B.

\subsection{Imaging Evaluation}

All patients had preoperative X-ray examination and MR examination. X-ray was reexamined on the first day after operation, followed up for 1 month, 3 months, half a year and 1 year. X-ray and MR were performed immediately in patients suspecting recurrent fracture symptoms.

\subsubsection{Imaging Evaluation}
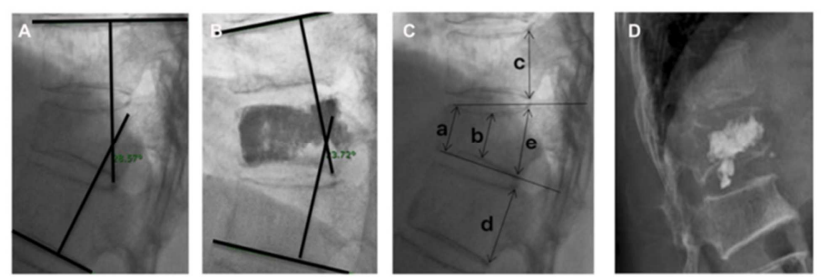

Figure 1. Imaging evaluation and measurement.

The correction angle of kyphosis is as follows (Figure 1A): The kyphotic angle of the vertebral body is defined as the vertical line of the respective extension lines of the upper endplate of the adjacent vertebral body above the OVCF vertebral body and the lower endplate of the lower adjacent vertebral body on the lateral X-ray film, and the angle formed by the two vertical lines was measured and recorded (Figure 1A and B). The difference between the two measurements was calculated as the correction angle of kyphosis. Degree of vertebral height recovery (Figure 1C): on lateral X-ray, the average of the posterior height of the upper and lower vertebral bodies adjacent to the OVCF was used to estimate the posterior vertebral height of OVCF (e is the estimated value (the average value of $\mathrm{c}$ and $\mathrm{d}$ ) of height of posterior vertebral body), and then the anterior edge of OVCF was measured ( $a$ is the anterior height of the vertebral body). The compression degree of vertebral anterior height $=\mathrm{a} / \mathrm{e}(\%)$. The recovery degree of anterior vertebral height was the difference between the preoperative and postoperative anterior vertebral height compression.

Bone cement leakage in intervertebral disc: observe whether there is bone cement leakage into intervertebral disc by X-ray film of vertebral (standard anteroposterior and lateral position) on the first day after operation (see Figure
1D).

\subsubsection{Pfirrmann Classification of Intervertebral Disc}

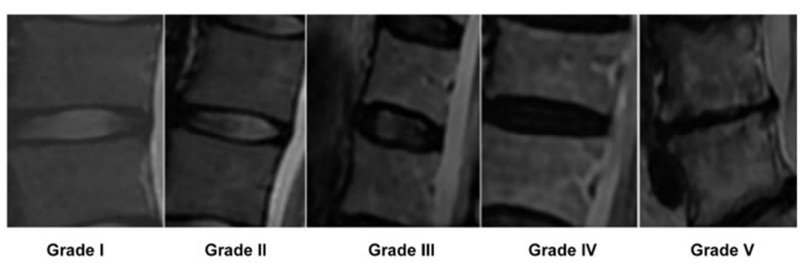

Figure 2. Pfirrmann [4] classification of intervertebral disc (5-grade method).

Grade 1: T2 weighted images showed homogeneous high signal of nucleus pulposus of intervertebral disc, which was the same as cerebrospinal fluid signal. MRI showed white images, which could clearly identify the nucleus pulposus and adjacent annulus fibrosus, also the intervertebral disc was of normal height; Grade 2: T2 weighted images showed homogeneous high signal of nucleus pulposus of intervertebral disc, which was the same as cerebrospinal fluid signal. MRI showed white images with dark color strip in nucleus pulposus, which could clearly identify nucleus pulposus and adjacent annulus fibrosus, also the intervertebral disc was of normal height; Grade 3: T2 weighted images showed low signal of nucleus pulposus, MRI showed gray images, and was unable to distinguish nucleus pulposus and adjacent annulus fibrosus, and the intervertebral disc height decreased; Grade 4: T2 weighted images showed that the signal of intervertebral disc was extremely low, MRI showed that the intervertebral disc was black, and was unable to distinguish the nucleus pulposus and adjacent annulus fibrosus, and the intervertebral disc height decreased significantly; Grade 5: T2 weighted images showed that the signal of intervertebral disc was extremely low with no homogeneous, MRI showed that the intervertebral disc was black, and was unable to distinguish the nucleus pulposus and adjacent annulus fibrosus, and the intervertebral space became significantly small. Among them, grade 1-2 were normal and grade 3 and above were degeneration [5].

\subsection{Observation Factors}

The age, gender, bone mineral density, kyphosis correction degree, vertebral height recovery degree, bone cement injection amount, Pfirrmann classification of intervertebral disc adjacent to injured vertebral and bone cement leakage in intervertebral disc were collected.

\subsection{Statistical Analysis}

The incidence of new fractures after PVP was analyzed through univariate and multivariate analysis to determine the related factors and risk factors. T-test was carried out for measurement data. The $\mathrm{X}^{2}$ test was used for counting data. Logistic regression analysis was used to evaluate the correlation between new OVCF and age, gender, 
preoperative basic disease, bone mineral density (T-score), bone cement injection volume, correction degree of kyphosis, recovery degree of vertebral height, Pfirrmann classification of intervertebral disc adjacent to injured vertebral and bone cement leakage in intervertebral disc. All the data were processed by SPSS 18.0 software. If $\mathrm{P}<0.05$, it was of statistically significance.

\section{Result}

A total of 401 patients were observed, including 308 females and 93 males, aged 50-99 years old (mean 74.4 years old). The follow-up time was 24-49 months (mean 28.5 months). There were 368 cases with 1 segment fracture, 30 cases with 2 segments fracture, 3 cases with more than 3 segments fracture. During the follow-up period, 34 patients $(34 / 401,8.4 \%)$ occurred new OVCF. New fracture was happened 1-49 months (average 8.6 months). Among them, $20.6 \%$ (7/34) new cases occurred within 3 months after PVP, $32.4 \%(11 / 34)$ within 6 months, and 50\% (17/34) within 12 months. In all OVCF patients treated for the first time, $\mathrm{T}$ value of BMD was $-2.9 \pm 1.0$, in which $-2.8 \pm 1.0$ in Group A and $-3.8 \pm 0.8$ in Group B. 27.2\% (109/401) Pfirrmann classification of intervertebral disc adjacent to injured vertebral was normal, of which $8.9 \%(3 / 34)$ in Group A and 28. $9 \%(106 / 367)$ in Group B. $72.8 \%$ (292/401) was classified as degeneration, of which $91.1 \%(31 / 34)$ in Group A and 71.1\% (261/367) in Group B. 11.5\% (46/401) of patients underwent first PVP occurred bone cement leakage in intervertebral disc, of which $67.6 \%(23 / 34)$ in Group A, and $6.3 \%(23 / 367)$ in Group B.

By univariate analysis, Pfirrmann classification in adjacent intervertebral disc of injured vertebral and bone cement leakage in intervertebral disc were found to be statistically significant $(\mathrm{P}<0.05)$ (Table 1). The potential risk factors with statistically significant difference confirmed by univariate analysis were substituted into Logistic regression analysis, and were furtherly performed multivariate analysis. Then we found that Pfirrmann classification of intervertebral disc adjacent to injured vertebral and bone cement leakage in intervertebral disc were significantly correlated with new vertebral fracture after PVP in the new fracture group. The risk of new OVCF increased by 3.732 times (95\% CI 1.107-12.581) when the adjacent intervertebral disc of injured vertebral was in degeneration. The risk of new OVCF increased by 31.818 times (95\% CI 13.384-75.640) when bone cement leakage occurred in injured vertebral (Table 2).

Table 1. Univariate Analysis of Group A and Group B.

\begin{tabular}{|c|c|c|c|}
\hline \multirow{2}{*}{ Risk factors of new fracture in vertebral } & \multicolumn{2}{|c|}{ Mean \pm SD or No. $(\%)$} & \multirow{2}{*}{$P$ value } \\
\hline & Group A $(n=34)$ & Group B $(n=367)$ & \\
\hline Gender (Female/male) & $25 / 9$ & $283 / 84$ & 0.636 \\
\hline Age & $75.4 \pm 7.4$ & $74.6 \pm 8.6$ & 0.970 \\
\hline Bone Mineral Density (BMD) & $-3.8 \pm 0.8$ & $-2.8 \pm 1.0$ & 0.261 \\
\hline PMMA Injected Volume (mL) & $5.1 \pm 1.8$ & $4.7 \pm 1.7$ & 0.067 \\
\hline Recovery Angel of Kyphosis $\left({ }^{\circ}\right)$ & $1.3 \pm 3.7$ & $1.8 \pm 5.9$ & 0.467 \\
\hline Recover Degree of Vertebral Height (\%) & $4.0 \pm 9.0$ & $8.0 \pm 11.1$ & 0.469 \\
\hline Bone Cement Leakage in Intervertebral Disc & $23(67.6)$ & $23(6.3)$ & $<0.001^{*}$ \\
\hline
\end{tabular}

Table 2. Multivariate Logistic Regression Equation Analysis.

\begin{tabular}{llll}
\hline Risk Factors & OR & P & 95\% CI for OR \\
\hline Pffirmann Classification of Intervertebral Disc (Degeneration) & 3.732 & $0.034^{*}$ \\
Leakage in Intervertebral Disc & 31.818 & $<0.001^{*}$ & $1.107-12.581$ \\
\hline
\end{tabular}

\section{Discussion}

At present, the incidence of new fractures after PVP in different studies is not consistent, which may be due to the different sample amount, sample characteristics, follow-up status, operation details and so on. Li et al. [6] reported that the incidence of new fractures in 166 patients after PVP was 38\%. However, Uppin et al. [7] reported that among 177 patients after PVP, 36 cases of new OVCF were occurred in 22 patients after treatment, with the incidence rate of $12.4 \%$. In this study, the incidence of new OVCF after PVP was 8.4\% (34/401).

Clinically, bone mineral density (BMD) is one of the main indicators reflecting the severity of osteoporosis. Yoo et al. [5] pointed out that the lower the $\mathrm{T}$ value of BMD measured before operation, the higher the probability of new vertebral fracture after PVP. At the same time, Zuo's study [8] believed that there was no correlation between new vertebral fractures and PVP treatment. Even conservative would have $25 \%$ chance of new vertebral fractures. In this study, all patients who underwent PVP surgery had a T value of less than -2.5 , and because they had a history of fragility fractures, they could be diagnosed as severe osteoporosis. Although osteoporosis is an important risk factor for OCVF in patients, for patients who have reached the diagnostic criteria of severe osteoporosis and underwent PVP treatment, the cause of recurrent fracture may be more related to other factors obviously, and a lower $\mathrm{T}$ value did not increase the risk of new fracture after surgery.

The current research results on whether the correction of kyphosis angle and the recovered vertebral height are related to the occurrence of refracture after PVP were different. Ma $X$ [9] and Rohlmann et al. [10] studies have shown that if the kyphosis deformity after PVP was not improved, it would 
increase the risk of recurring fracture. However, Kim et al. [11] believe that the greater the recovery degree of injured vertebral height during PVP, the higher risk of new fracture in adjacent vertebral. The study of Christopher et al. [12] shows that the probability of new fracture in adjacent vertebral body increases by $9 \%$ every $1^{\circ}$ correction of local kyphosis angle of injured vertebral body. It can be inferred that the biomechanical changes of the spine are important factors for the new OVCF after PVP [13]. On the one hand, the maximum correction of kyphosis angle and the restoration of vertebral height can make the normal stress line of spine recover to the maximum extent, and the stress of each vertebral body is evenly distributed to avoid local stress concentration, thus reducing the occurrence of refracture [14]. However, when correcting kyphosis angle and restoring vertebral height, external force must be exerted on injured vertebral, the impact was especially bigger for patients with severe osteoporosis, which may be counterproductive. What kind of impact will be produced and how to benefit patients remain further research and confirmation? The result of this study is that there is no correlation between the correction of convex angle and the recovery of vertebral height and the occurrence of refracture after PVP.

At present, in PVP surgery, the optimal amount of bone cement injection is controversial. On the one hand, in order to ensure the curative efficacy of operation and avoid refracture of strengthened vertebral body, it was generally advocated in the clinical appliance to inject bone cement as enough as possible to enhance the strength of vertebral body. On the other hand, some scholars believe that if bone cement is injected excessively, it not only will easily cause leakage accident, but also strengthen the vertebral excessively and increase the strength difference between strengthened vertebral body and adjacent vertebral body, which will cause uneven distribution and excessive concentration of stress on vertebral body, and thus increase the risk of fracture of adjacent vertebral body $[15,16]$. However, in a prospective randomized controlled study by Yi et al. [17], it was found that there was no correlation between the amount of bone cement injection and the new vertebral fracture after PVP, which was consistent with the results of this study. Due to the limitations of sample and statistical methods, it is still not clear whether the recurrent of fracture in adjacent vertebral is caused by the natural course of osteoporosis or related to the excessive strengthening of injured vertebral.

In the spinal structural unit, intervertebral disc plays an important role in stress dispersion. The stiffness and hardness of degenerative intervertebral disc, nucleus pulposus and annulus fibrosus are increased, and the buffering capacity is weakened, which has an impact on the stress distribution and load transfer of adjacent vertebral bodies and even the whole spine [18, 19]. Hansson [20] study found that the risk of OCVF was increased in elderly people with Pfirrmann classification of intervertebral disc as degeneration. Kosmopoulos et al. [21] evaluated the changes in the von Mises pressure distribution of the vertebral body with cement-strengthened vertebral bodies in two models of degenerative and normal grading. In the model of intervertebral disc degeneration, the pressure load on the strengthened vertebral body and adjacent vertebral bodies had been transferred, thereof the pressure load of the upper and lower endplates of the annulus fibrosus increased, while the pressure load of the central endplate of the nucleus pulposus decreased. After studying the relevant finite element model, Baroud et al. [22] confirmed that the pressure of adjacent intervertebral discs increased by $19 \%$ after PVP. Thus, a vicious circle process of "intervertebral disc degeneration -> vertebral fracture $->$ accelerating intervertebral disc degeneration -> recurrent vertebral fracture" is produced. At the same time, bone cement leakage is one of the common complications of PVP surgery, and the bone cement leakage of intervertebral disc damages the structure of intervertebral disc, promotes the progress of intervertebral disc degeneration, and forms column pier effect, which leads to the weakening of intervertebral disc buffer function, changes the stress of adjacent vertebral endplate, and increases the risk of recurrent fracture [18]. Sun et al. [23] carried out a retrospective analysis on cases of bone cement leakage in intervertebral disc, and found that the chance of new fracture of adjacent vertebral body was 44\%, which was 6 times higher than that of cases without bone cement leakage. Lin et al. [24] retrospectively studied and found that the incidence of new fractures of adjacent vertebral bodies was $58 \%$ in patients with bone cement leakage in intervertebral discs, while the incidence of new fractures in patients without bone cement leakage was only $12 \%$. These results are consistent with this study. Therefore, to protect the intervertebral disc and slow down its degeneration speed should be able to effectively reduce the risk of vertebral recurrent fracture, especially to avoid the leakage of bone cement in intervertebral disc during the operation. More and more clinicians pay more attention to this, and are committed to the research and improvement of surgical techniques and details to reduce the leakage rate of bone cement.

\section{Conclusion}

(1) The incidence of recurrent vertebral fracture after PVP was $8.4 \%, 50 \%$ of which occurred within 12 months after PVP. (2) There was no significant correlation in gender, age, bone mineral density, the amount of bone cement injected, the degree of kyphosis correction and the degree of vertebral height recovery with new fracture of vertebral after PVP. (3) Pfirrmann classification of intervertebral disc adjacent to hurt verterbral and bone cement leakage in intervertebral disc were significantly correlated with the occurrence of vertebral new fracture after PVP. (4) In PVP operation, the leakage of bone cement should be avoided as far as possible in order to reduce the recurrence of vertebral compression fractures.

\section{References}

[1] H. Tang, J. Zhao, and C. Hao. Osteoporotic vertebral compression fractures: surgery versus non-operative management. J Int Med Res, 2011, 39 (4): 1438-47. 
[2] Y. J. Rho, W. J. Choe, and Y. I. Chun. Risk factors predicting the new symptomatic vertebral compression fractures after percutaneous vertebroplasty or kyphoplasty. Eur Spine J, 2012, 21 (5): 905-11.

[3] A. T. Trout and D. F. Kallmes. Does vertebroplasty cause incident vertebral fractures? A review of available data. AJNR Am J Neuroradiol, 2006, 27 (7): 1397-403.

[4] C. W. Pfirrmann, A. Metzdorf, M. Zanetti, J. Hodler, and N. Boos. Magnetic resonance classification of lumbar intervertebral disc degeneration. Spine (Phila Pa 1976), 2001, 26 (17): 1873-8.

[5] C. M. Yoo, K. B. Park, S. H. Hwang, D. H. Kang, J. M. Jung, and I. S. Park. The analysis of patterns and risk factors of newly developed vertebral compression fractures after percutaneous vertebroplasty. J Korean Neurosurg Soc, 2012, 52 (4): 339-45.

[6] Y. A. Li, C. L. Lin, M. C. Chang, C. L. Liu, T. H. Chen, and S. C. Lai. Subsequent vertebral fracture after vertebroplasty: incidence and analysis of risk factors. Spine (Phila $\mathrm{Pa}$ 1976), 2012, 37 (3): 179-83

[7] A. A. Uppin, J. A. Hirsch, L. V. Centenera, B. A. Pfiefer, A. G. Pazianos, and I. S. Choi. Occurrence of new vertebral body fracture after percutaneous vertebroplasty in patients with osteoporosis. Radiology, 2003, 226 (1): 119-24.

[8] J. Zou, X. Mei, X. Zhu, Q. Shi, and H. Yang. The long-term incidence of subsequent vertebral body fracture after vertebral augmentation therapy: a systemic review and meta-analysis. Pain Physician, 2012, 15 (4): E515-22.

[9] X. Ma, D. Xing, J. Ma, J. Wang, Y. Chen, W. Xu, Y. Yang, B. $\mathrm{Ma}$, and S. Zhu. Risk factors for new vertebral compression fractures after percutaneous vertebroplasty: qualitative evidence synthesized from a systematic review. Spine (Phila Pa 1976), 2013, 38 (12): E713-22.

[10] A. Rohlmann, H. N. Boustani, G. Bergmann, and T. Zander. A probabilistic finite element analysis of the stresses in the augmented vertebral body after vertebroplasty. Eur Spine J, 2010, 19 (9): 1585-95.

[11] S. H. Kim, H. S. Kang, J. A. Choi, and J. M. Ahn. Risk factors of new compression fractures in adjacent vertebrae after percutaneous vertebroplasty. Acta Radiol, 2004, 45 (4): 440-5.

[12] C. Bliemel, L. Oberkircher, B. Buecking, N. Timmesfeld, S. Ruchholtz, and A. Krueger. Higher incidence of new vertebral fractures following percutaneous vertebroplasty and kyphoplasty--fact or fiction? Acta Orthop Belg, 2012, 78 (2): 220-9.

[13] I. Movrin, R. Vengust, and R. Komadina. Adjacent vertebral fractures after percutaneous vertebral augmentation of osteoporotic vertebral compression fracture: a comparison of balloon kyphoplasty and vertebroplasty. Arch Orthop Trauma Surg, 2010, 130 (9): 1157-66.

[14] T. J. Kaufmann, A. T. Trout, and D. F. Kallmes. The effects of cement volume on clinical outcomes of percutaneous vertebroplasty. AJNR Am J Neuroradiol, 2006, 27 (9): 1933-7.

[15] C. H. Yen, M. M. Teng, W. H. Yuan, Y. C. Sun, and C. Y. Chang. Preventive vertebroplasty for adjacent vertebral bodies: a good solution to reduce adjacent vertebral fracture after percutaneous vertebroplasty. AJNR Am J Neuroradiol, 2012, 33 (5): 826-32.

[16] A. S. Mudano, J. Bian, J. U. Cope, J. R. Curtis, T. P. Gross, J. J. Allison, Y. Kim, D. Briggs, M. E. Melton, J. Xi, and K. G. Saag. Vertebroplasty and kyphoplasty are associated with an increased risk of secondary vertebral compression fractures: a population-based cohort study. Osteoporos Int, 2009, 20 (5): 819-26.

[17] X. Yi, H. Lu, F. Tian, Y. Wang, C. Li, H. Liu, X. Liu, and H. Li. Recompression in new levels after percutaneous vertebroplasty and kyphoplasty compared with conservative treatment. Arch Orthop Trauma Surg, 2014, 134 (1): 21-30.

[18] E. Ibarz, A. Herrera, Y. Mas, J. Rodriguez-Vela, J. Cegonino, S. Puertolas, and L. Gracia. Development and kinematic verification of a finite element model for the lumbar spine: application to disc degeneration. Biomed Res Int, 2013, 2013: 705185 .

[19] N. Inoue and A. A. Espinoza Orias. Biomechanics of intervertebral disk degeneration. Orthop Clin North Am, 2011, 42 (4): 487-99, vii.

[20] T. Hansson and B. Roos. The relation between bone mineral content, experimental compression fractures, and disc degeneration in lumbar vertebrae. Spine (Phila Pa 1976), 1981, $6(2): 147-53$.

[21] V. Kosmopoulos, T. S. Keller, and C. Schizas. Early stage disc degeneration does not have an appreciable affect on stiffness and load transfer following vertebroplasty and kyphoplasty. Eur Spine J, 2009, 18 (1): 59-68.

[22] G. Baroud, J. Nemes, P. Heini, and T. Steffen. Load shift of the intervertebral disc after a vertebroplasty: a finite-element study. Eur Spine J, 2003, 12 (4): 421-6.

[23] Y. C. Sun, M. M. Teng, W. S. Yuan, C. B. Luo, F. C. Chang, J. F. Lirng, W. Y. Guo, and C. Y. Chang. Risk of post-vertebroplasty fracture in adjacent vertebral bodies appears correlated with the morphologic extent of bone cement. J Chin Med Assoc, 2011, 74 (8): 357-62.

[24] J. Lin, L. Zhang, and H. L. Yang. Unilateral versus bilateral balloon kyphoplasty for osteoporotic vertebral compression fractures. Pain Physician, 2013, 16 (5): 447-53. 